\title{
The impact of daily text message reminders on medication adherence in healthy volunteers and patients with coronary artery disease
}

\author{
Avinash Kumar Pandey
}

\begin{abstract}
AK Pandey. The impact of daily text message reminders on medication adherence in healthy volunteers and patients with coronary artery disease. Curr Res Cardiol 2015;2(1):19-22.

BACKGROUND: Rates of medication nonadherence are high, which can lead to disease progression, disability and death.

OBJECTIVE: To test a potential solution to improve adherence to medications using a novel computer-based text message reminder system. METHODS: An automated computer program to send text messages to cell phones was developed. In phase 1, the reliability of the system was tested by sending text messages four times daily for two months to four volunteers. In phase 2, 20 healthy volunteers were asked to take a placebo at four prespecified times per day for one week with text message reminders and one week without. In Phase 3, 30 random, stable cardiac patients were recruited and instructed to take and record medications according to their own regimen for one month with text message reminders and one month without.
\end{abstract}

A edication nonadherence or noncompliance is defined as the I incorrect taking of medications, whether in incorrect quantity or incorrect time as prescribed (1-6). This can limit the effectiveness of medications, lead to disease progression and, in some extreme cases, lead to premature death $(5,6)$. Studies suggest that medication nonadherence is extremely prevalent (1-6).

Nonadherence to medication is categorized into two main groups: intentional and nonintentional (7). Intentional nonadherence can include deliberately choosing not to take medications due to personal preference, not understanding or believing in the importance of the medication, or choosing not to take the medication due to perceived side effects (7). Nonintentional nonadherence occurs when the individual is not deliberately refusing to take medications but may be making medication errors unintentionally due to factors such as complexity of medication regimens, inability to acquire or administer the medication, or forgetting to take the medication (7).

Studies have identified forgetfulness as a significant contributor to medication nonadherence $(4,6,7)$. Although systems exist today to reduce nonadherence, few focus on reducing forgetfulness - a nonintentional form of nonadherence $(3,4)$. The systems that do address forgetfulness often are only able to address one part of forgetfulness: beepers remind patients to take medications but do not inform them of what medications to take. Pill boxes help patients to take the correct medications, but do not remind them to take them. In addition, because carrying these systems is cumbersome, they are often left at home, thereby reducing their effectiveness $(3,4)$. Certain populations, such as elderly individuals, those with cognitive or neurological diseases, such as stroke, dementia and depression, as well as those with lower educational levels, have been shown to be at greater risk for medication nonadherence $(1,3,5,8)$. Available systems, such as pill boxes, blister packs and beepers, have had limited success at reducing nonadherence (relative risk reduction of only $5 \%$ to 20\% ) (4-6). An effective solution to this clinical problem has yet to be identified (4).
RESULTS: In phase 1, the system accurately delivered $100 \%$ of reminder messages. In phase 2 , the adherence to placebos improved with text message reminders in $88 \%$ of healthy volunteers, with a $65 \%$ relative risk reduction for nonadherence $(\mathrm{P}<0.01)$. In phase 3 , adherence to medication with text message reminder improved in $100 \%$ of cardiac patients, with a $64 \%$ relative risk reduction for nonadherence $(\mathrm{P}<0.01)$. In all subgroups analyzed, text message reminders significantly improved medication adherence.

CONCLUSIONS: The system proved to be effective in improving adherence to placebo in healthy volunteers and to medications in cardiac patients over a two-month period. Further research is required to assess the effects of this system over longer periods of time and in more diverse populations.

Key Words: E-solution; Medication adherence; Placebo; Reminder system

A more effective, automated system of reminders could be used to help reduce forgetfulness-induced nonadherence to medication.

The present study tested the feasibility and the efficacy of a novel, computerized, automated text message (SMS) system to decrease nonadherence to placebos and medications.

Design

\section{METHODS}

The present study was a single-centre, pre-/postintervention analysis of medication adherence using SMS reminders. There were three phases to the present study: phase 1 - technology pilot test; phase 2 - pilot testing in volunteers; and phase 3 - real-world intervention.

The study comprised three phases with two initial pilot phases to ensure the feasibility and technical reliability of the technology and its ability to improve adherence to placebo, followed by a real-world assessment involving volunteer cardiac patients from a community cardiology clinic using their own medications taken according to their own regimen.

\section{Ethics}

A test protocol and written informed consent form were reviewed by the University of Waterloo Human Research Ethics Board (Waterloo, Ontario). Written informed consent was obtained from all participants before any study procedures were performed.

\section{Setting}

The present study was performed in Waterloo (population 100,000) and Cambridge (population 150,000), Ontario. Recruitment of four volunteers to test the reliability of the system occurred in Waterloo. Recruitment of 25 healthy volunteers for a pilot test also occurred in Waterloo. Thirty cardiac patients were recruited at Cambridge Cardiac Care Centre, a community cardiology clinic serving Waterloo region (population 550,000).

Center for Healthcare Delivery Sciences, Brigham and Women's Hospital, Harvard Medical School; Cambridge Cardiac Care Centre, Cambridge, Ontario Correspondence and reprints: Avinash K Pandey, Cambridge Cardiac Care Centre, 150 Hespeler Road, Cambridge, Ontario N1R 6 V6.

Telephone 519-624-3511, fax 519-624-3411, e-mail avinash@child2child.ca 
TABLE 1

Phase 2: healthy volunteer pilot. Adherence to placebo in healthy volunteers measured by logbook

\begin{tabular}{lccc}
\hline Healthy volunteers & $\begin{array}{c}\text { Without } \\
\text { reminders }\end{array}$ & $\begin{array}{c}\text { With } \\
\text { reminders }\end{array}$ & P \\
\hline Total missed doses, $\mathrm{n}$ & 155 & 67 & $<0.01$ \\
Missed doses per person, mean & 9.7 & 4.2 & $<0.01$ \\
Percentage of time doses were taken & 65.4 & 85.0 & $<0.01$ \\
Individuals who improved with reminders, n & N/A & 14 & N/A \\
Individuals who improved with reminders, \% & N/A & 87.5 & N/A \\
Absolute improvement in adherence, \% & N/A & 19.6 & $<0.01$ \\
Relative improvement in adherence (relative & N/A & 64.8 & $<0.01$ \\
$\quad$ risk reduction for nonadherence), \% & & & \\
\hline N/A Not applicable & & &
\end{tabular}

\section{Population and subject selection}

In phases 1 and 2, the populations studied were healthy volunteers. All participants were adults who had cell phones. In phase 3, the population comprised volunteers from a Canadian cardiology community practice (Cambridge Cardiac Care Centre). The study population size was determined for phase 3 through power analysis of phase 2 data. All stable cardiac patients $>18$ years of age who were able to read and write English and competent to provide informed consent were included. Children, individuals without a cell phone, or who were unable to read and write in English, unable to provide informed consent, unable to comply with study protocol and incarcerated individuals were excluded from the study.

\section{Phase 1: technology pilot}

In phase 1, a computer program was created to send text messages to cell phones using Microsoft Visual Basic 2008 Express Edition (Microsoft Corporation, USA). The SMS reminder system checks the time every minute until it reaches a specific time when a patient is required to take a medication. The system sends an e-mail to a web portal that converts the e-mail to a text message and sends it to the patient's cell phone. The web portal system also sends an e-mail to an audit trail e-mail account that can be used to ensure appropriate delivery of the text messages. Text messages were sent four times daily to four volunteers with different cell phones and different cell phone providers over a two-month period to validate the stability and reliability of this system. Volunteers were asked to keep their cell phones charged and turned on throughout this two-month system stability assessment. Participants were asked to log (in supplied notebooks) the time of receipt of the text message when it arrived on their cell phones.

\section{Phase 2: healthy volunteer pilot}

Twenty-five healthy volunteers were recruited to test this model. Demographic information was obtained on all volunteers: age, sex, education level, cell phone number and cell phone provider. The test procedure involved self-reported removal of a placebo (a raisin) from vials. Individuals had to remove and discard a raisin at 07:30, 12:00, 18:00 and 21:00. Volunteers were randomly assigned, using a web based random number generator (www.randomnumbergenerator.com), to receive text message reminders in either week one or week two. For one week individuals removed the placebo raisins without any reminders. For a second week, they received reminder text messages and had to record whether they received the text message and whether they removed the raisin. Volunteers recorded removal of raisins and receipt of text messages in specially prepared and supplied logbooks. One-half of the subjects began receiving text message reminders for the first week and received no text message for the second week, while the other onehalf of subjects did not received text message reminders in the first week, and received text message reminders in the second week. Adherence was determined by measuring the number of total prescribed doses of raisins, and the number of doses of placebos actually removed as recorded in log books. Total overall accuracy of selfreported placebo removal was determined through a 'pill count' of returned vials of raisins. Individuals had been randomly assigned to receive text messages either in week one or week two to mitigate a potential trainer effect bias (a bias introduced by being exposed to a specific task and becoming familiar with the task such that it influences the result of a subsequent intervention being investigated) (9).

\section{Phase 3: real-world validation}

Thirty random volunteers were recruited from a local adult cardiology practice. Written informed consent was obtained. Demographic information was obtained for all volunteers. Volunteers were asked to take their own medications at their own prescribed regimen for one month and record their adherence in a specially designed logbook. For a second month, they received reminder text messages and were asked to record their adherence according to their own prescribed regimen. The percent adherence was determined by dividing the medications taken by the medication prescribed. One-half of the subjects were randomly assigned to receive text message reminders for the first month and received no text message for the second month, while the other one-half of subjects were randomized not to receive text message reminders in the first month and to receive text message reminders in the second month, to mitigate a potential trainer effect.

\section{Data analysis}

Three main types of analysis were performed: absolute risk reduction, relative risk reduction and subgroup analysis. Adherence was determined from patient medication logs by calculating the number of total prescribed doses and measuring the number of doses actually taken as recorded in log books (9). Absolute risk reduction was determined by comparing average adherence of individuals receiving reminders with that of individuals not receiving reminders. Relative risk reduction was determined by dividing the absolute risk reduction by the control incidence rate (nonadherence in the individuals not receiving reminders). Certain groups, chosen prospectively according to literature review were identified as having a greater risk of medication regimen nonadherence (elderly, less than grade 12 education, depression and dementia). Adherence rates and the impact of the text message reminder system in these prespecified groups was compared with the total study population.

\section{Statistical analysis}

GraphPad Instat 3.10 (GraphPad, USA) was used to perform statistical analysis. Paired Student's $t$ tests were used for comparisons of two variables because the same subjects participated in each period of time and served as their own controls. ANOVAs with Bonferroni correction tests (for multiple comparisons) were used for comparisons with $\geq 2$ variables; $\mathrm{P}<0.05$ was considered to be statistically significant.

Phase 1

\section{RESULTS}

In the first phase of the study (the feasibility pilot), four healthy volunteers received and logged 100\% of text messages sent. Each text message reminder logged by the volunteers was verified with an e-mail audit trail. All messages were sent in a timely and accurate fashion.

\section{Phase 2}

Table 1 presents the results from the cohort study in phase 2. A high rate of nonadherence to placebo removal was noted in healthy volunteers (35\%). Improvements in adherence with the SMS system were consistent, with an average absolute improvement of $20 \%$ and a relative risk reduction for nonadherence of $65 \%$. Text message reminders were reliably sent from an automated computer program and were effective at improving adherence in all groups studied. Adherence was determined using self-recorded logbooks, a commonly used method to determine medication adherence (8). Whether volunteers received 
TABLE 2

Phase 3: real-world validation. Adherence to routine medications in stable cardiac patients measured by logbook

\begin{tabular}{lccc}
\hline Stable cardiac patients & $\begin{array}{c}\text { Without } \\
\text { reminders }\end{array}$ & $\begin{array}{c}\text { With } \\
\text { reminders }\end{array}$ & P \\
\hline Prescribed doses per month, $\mathrm{n}$ & 2016 & 2016 & N/A \\
Correctly taken doses per month, n & 1705 & 1897 & $<0.01$ \\
Prescribed doses per week, mean & 67.2 & 67.2 & N/A \\
Doses taken for the week per person, mean & 56.8 & 63.2 & $<0.01$ \\
Percent taken correctly & 83.0 & 93.8 & $<0.01$ \\
Improved subjects, \% & N/A & 100 & N/A \\
Absolute improvement in adherence, \% & N/A & 10.8 & $<0.01$ \\
Relative improvement in adherence (relative & N/A & $63.9 \%$ & $<0.01$
\end{tabular}

risk reduction for nonadherence), \%

N/A Not applicable

text message reminders in the first half or second half of the study did not affect adherence rates, suggesting no significant trainer effect in healthy volunteers in the present study.

Phase 3

Table 2 shows the results from phase 3 the cohort study of random cardiac patients taking their own medications according to their own regimen with or without a text message reminder. A high rate of medication nonadherence was noted in cardiac patients (17\%). This system of text message reminders consistently improved adherence to medications, with all subjects improving. The average absolute improvement was $11 \%$, with a relative risk reduction for nonadherence of $64 \%$. Certain groups of cardiac patients appeared to have lower baseline adherence. The elderly, stroke, dementia and depression patients as well as those with the less education showed lower rates of adherence to medications (Table 3). Whether individuals received text message in the first half of the study or in the second half of the study did not impact the adherence rates significantly in any groups. As a result, no trainer effect was noted in these cardiac patients.

\section{Subgroup analysis}

Table 3 illustrates the adherence rates of specific subgroups prospectively selected who may be at higher risk of nonadherence to medications. As previously noted, stroke, depression, dementia and elderly patients were indeed found to exhibit less adherent to medications (74\% adherence rate on average compared with the total study population adherence rate of $83 \%$ ). All these subgroups with higher nonadherence rates also appeared to benefit more than the overall study population suggesting this system may be particularly efficacious in those at highest risk for nonadherence (on average, 18\% absolute improvement in adherence to medications in these groups with higher baseline nonadherence compared with the total study population improvement of $11 \%[\mathrm{P}<0.05])$.

\section{Stratification according to education}

Figure 1 illustrates the improvements of patients stratified according to education level. Previous studies have noted that lower levels of education is a predictor for nonadherence to medications. It was noted in the present study that individuals with fewer years of education (having attained $\leq 12$ years of education) had lower baseline adherence but also appeared to improve adherence more through this text message reminder system (an 18\% improvement versus 11\% in the total study population $[\mathrm{P}<0.05])$.

\section{Reasons for nonadherence}

Figure 2 shows the participant self-perceived reasons for nonadherence to their own medications. Forgetfulness represented $71 \%$ of the reasons identified by the participants for nonadherence. Cost, side effects
TABLE 3

Phase 3: subgroup analysis. Adherence to routine medications in stable cardiac patients in certain subgroups previously identified based on literature review to be at higher risk for inactivity as well as stratified according to education

\begin{tabular}{lccc}
\hline Group & $\begin{array}{c}\text { Without } \\
\text { reminders }\end{array}$ & $\begin{array}{c}\text { With } \\
\text { reminders }\end{array}$ & P \\
\hline All subjects & 83.0 & 93.8 & $<0.01$ \\
Stroke & 68.2 & 88.6 & $<0.05$ \\
Dementia & 79.9 & 94.2 & $<0.01$ \\
Depression & 73.8 & 91.8 & $<0.01$ \\
Elderly (>65 years of age) & 84.0 & 96.1 & $<0.01$ \\
Extreme elderly (>80 years of age) & 65.4 & 89.5 & $<0.05$ \\
<Grade 12 education & 75.0 & 93.8 & $<0.01$ \\
Grade 12 education & 84.3 & 94.2 & $<0.05$ \\
Postsecondary education & 85.2 & 94.8 & $<0.01$ \\
\hline
\end{tabular}

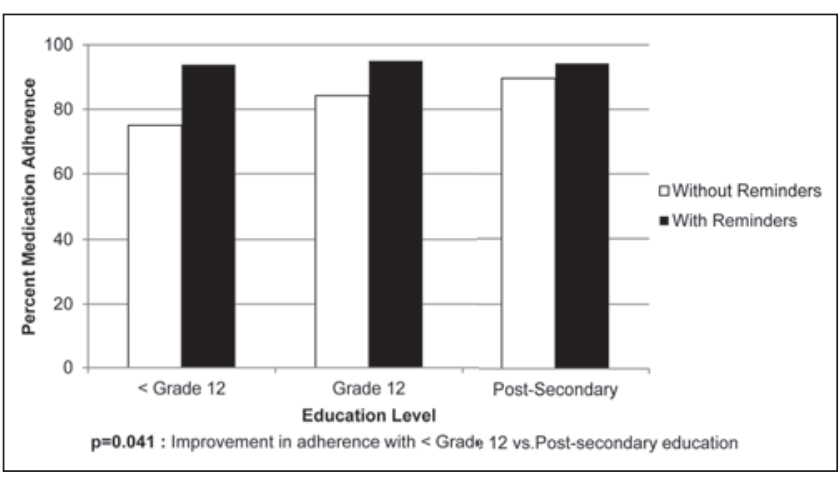

Figure 1) Improvement in adherence to medications with and without text message reminders stratified according to education level. Individuals with less than grade 12 education improved on average 19\% with text message reminders. Individuals with a grade 12 education improved, on average, $11 \%$ with text message reminders. Individuals with post-secondary education improved on average $6 \%$ with text message reminders. High rates of medication nonadherence were found in those with less education but these individuals were also found to improve more with text message reminders

and routine were also reasons that participants believed caused them to be nonadherent to their medications.

\section{DISCUSSION}

An absolute improvement in adherence of $>5 \%$ can be considered to represent a clinically significant improvement (5). In a population of stable cardiac patients, the present study demonstrated an $11 \%$ absolute improvement in medication adherence, suggesting that this system may have a meaningful clinical impact. This is in accordance with studies that have demonstrated the ability of communication technology to be used as a motivator for improving adherence to medications (1). The present study is the first, however, to test the use of text messages as a mode of medication reminder for reducing forgetfulness.

Subgroups identified in previous studies to be at higher risk for nonadherence to medication were demonstrated to be less adherent to medications in the present study. However, these groups were demonstrated to improve more with this system, perhaps as a result of their increased risk for forgetfulness. A lower education level has been previously linked to lower medication adherence. In all groups analyzed, individuals with lower baseline adherence rates appeared to benefit most from this system

While this system is designed to address only forgetfulness as a cause of nonadherence, it appears to have a marked effect on reducing nonadherence, with $100 \%$ of cardiac patients demonstrating some 


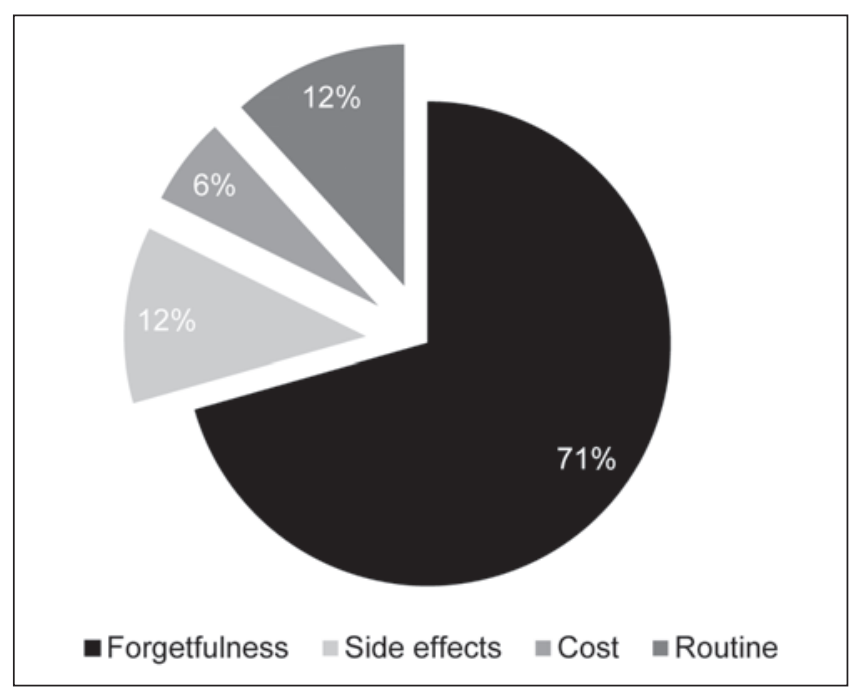

Figure 2) Participant self-perceived reasons for nonadherence. A total of $71 \%$ of participants identified forgetfulness as a contributor to their nonadherence; $12 \%$ identified their daily routines as an impediment to their medication adherence; $6 \%$ of patients identified cost as a contributor to nonadherence; and $12 \%$ identified side effects as a contributor to their nonadherence. The text message reminder system may have been effective at improving adherence because it may help to alleviate forgetfulness, which is an important contributor to nonadherence in this population

degree of improvement in adherence with an average relative risk reduction for nonadherence of $64 \%$ in this population. This suggests that forgetfulness may be an important mechanism leading to medication nonadherence and strategies, such as this system, that effectively address forgetfulness may improve adherence to medications.

\section{Study limitations}

Not all individuals have cell phones; a preliminary test of this program, however, demonstrates that it is able to deliver a recorded voice message over landline telephones, which may mitigate this limitation. The present study included a small group of volunteers and was short in duration, measuring adherence over only a few months. A longerterm study assessing its impacts in high-risk post-myocardial infarction patients has been undertaken. Adherence in both groups within the study may have been artificially inflated due to the intervention of logbooks used to assess adherence. The present analysis was a selfreported adherence study, although past research has shown this to be an effective way to determine adherence (9).

\section{REFERENCES}

1. Friedman RH, Kazis LE, Jette A, et al. A telecommunications system for monitoring and counselling patients with hypertensionImpact on medication adherence and blood pressure control. Am J Hypertens 1996;9:285-92.

2. Piette JD, Weinberger M, McPhee SJ, et al. Do automated calls with nurse follow-up improve self care and glycemic control among vulnerable patients with diabetes? Am J Med 2000;108:20-7.
Future directions

In the future, a similar automated text message reminder system could be used to reduce medication nonadherence. The system could be offered by hospitals or clinics as a way of reducing nonadherence to medications. The applicability of the system could also be tested in numerous other fields to test its effectiveness at reducing nonadherence to lifestyle changes and exercise regimens. Strategies, such as this SMS reminder system, that effectively address forgetfulness may be expected to significantly impact nonadherence and, perhaps, over time contribute to a reduction in morbidity and mortality through greater adherence to appropriate medications.

This novel text message reminder system demonstrated reliability, accurately sending text messages to numerous cell phones with different cell phone providers without fail. In all groups studied, this system proved effective at reducing the number of missed doses, and appeared to be most effective in groups with lower baseline adherence.

ACKNOWLEDGMENTS: The author thanks Dr N Choudhry, Associate Professor, Harvard Medical School (Boston, Massachusetts, USA), Division of Pharmacoepidemiology and Pharmacoeconomics, Department of Medicine, Brigham and Women's Hospital (Boston, Massachusetts, USA) for his valuable discussions, and assistance with analysis and manuscript preparation; Dr Alter, cardiologist and associate professor in the Department of Medicine at the University of Toronto (Toronto, Ontario) for his useful discussions and assistance in manuscript preparation; Dr S Shankar, statistician at the University of Waterloo (Waterloo, Ontario) for her valuable aid in statistical analysis; Dr Susan Sykes, University of Waterloo Human Research Ethics Board for ethics oversight; and Ms Kelly Cronin, cardiac nurse, for aid with data collection. Furthermore, the author thanks the 59 volunteers who participated in this study.

SOURCES OF FUNDING: Neither the author nor the participants received any funding or compensation from any institution, public or private.

DISCLOSURE: A relative of the author is employed at the Cambridge Cardiac Care Centre, where this study was performed.

3. Rosen MI, Rigsby MO, Salahi JT, et al. Electronic monitoring and counseling to improve medication compliance. Behav Res Ther 2004:42:409-422.

4. Cutrona SL, Choudhry NK, Fischer MA, et al. Modes of delivery for interventions to improve cardiovascular medication adherence. Am J Manag Care 2010;16:929-42.

5. Choudhry NK, Avorn J, et al. Full coverage for preventive medications after myocardial infarction. N Engl J Med 2011;365:2088-97.

6. Rasmussen JN, Chong A, Alter DA. Relationship between adherence to evidence-based pharmacotherapy and long-term mortality after acute myocardial infarction. JAMA 2007;297:177-86.

7. Wroe AL. Intentional and unintentional nonadherence: A study of decision making. J Behav Med 2002;25:355-72.

8. MacLaughlin EJ, Raehl CL, Treadway AK, Sterling TL, Zoller DP, Bond CA. Assessing medication adherence in the elderly. Drugs Aging 2005;22:231-55.

9. Viera AJ, Bangdiwala SI. Eliminating bias in randomized controlled trials: Importance of Allocation concealment and masking. Fam Med 2007;39:132 